\title{
Probing of ultrahigh optical $Q$-factors of individual liquid microdroplets on superhydrophobic surfaces using tapered optical fiber waveguides
}

\author{
Alexandr Jonáš, ${ }^{1,3}$ Yasin Karadag, ${ }^{1}$ Michael Mestre, ${ }^{1,2}$ and Alper Kiraz ${ }^{1,4}$ \\ ${ }^{1}$ Department of Physics, Koç University, Rumelifeneri Yolu, Sariyer, Istanbul 34450, Turkey \\ ${ }^{2}$ Institut d'Optique, LP2N, Talence F-33405, France \\ ${ }^{3} e$-mail: ajonas@ku.edu.tr \\ ${ }^{4} e$-mail: akiraz@ku.edu.tr
}

Received July 19, 2012; revised October 5, 2012; accepted October 9, 2012;

posted October 10, 2012 (Doc. ID 172327); published November 8, 2012

\begin{abstract}
We report measurements of ultrahigh quality factors ( $Q$-factors) of the optical whispering-gallery modes excited via a tapered optical-fiber waveguide in single glycerol-water microdroplets standing on a superhydrophobic surface in air. Owing to the high contact angle of the glycerol-water mixture on the superhydrophobic surface $\left(>155^{\circ}\right)$, microdroplets with the geometry of a truncated sphere minimally distorted by gravity and contact line pinning effects could be generated. $Q$-factors up to $2.3 \times 10^{6}$ were observed for such droplets with radii of $100-200 \mu \mathrm{m}$ exposed to the ambient atmosphere in a closed chamber with controlled relative humidity. Placement of microdroplets in a constant humidity environment permitted prolonged characterization of $Q$-factors for individual microdroplets. We found that the $Q$-factors in air were stable over more than $1 \mathrm{~h}$ and their measured values were limited mostly by the thermally induced droplet shape fluctuations. (c) 2012 Optical Society of America OCIS codes: $\quad 140.3945,060.2340,130.3120,300.6320$
\end{abstract}

\section{INTRODUCTION}

Ultrahigh-quality (ultrahigh-Q) optical resonant microcavities that confine and concentrate light to small modal volumes enable optical fields to interact strongly with dipolar emitters embedded in the cavity or with mechanical degrees of freedom of the cavity [1]. Moreover, the spectral positions of their optical resonances are very sensitive to changes in the shape and/or composition of the cavity or its environment. These properties make ultrahigh- $Q$ microcavities ideally suited for applications in cavity QED, cavity opto-mechanics, and label-free biological sensing [1-4] .

The $Q$-factor of an optical resonant mode can be defined as

$$
Q=\frac{\omega}{\delta \omega}=\frac{\lambda}{\delta \lambda},
$$

where $\omega(\lambda)$ is the resonant frequency (wavelength) and $\delta \omega(\delta \lambda)$ is the resonance linewidth. Among various optical microcavities, such as micropillars, microdiscs, or photonic crystal defect cavities, microspheres and microtoroids display the highest- $Q$ optical resonances-so-called whispering gallery modes (WGMs) [1,5]. In order to excite such ultrahigh- $Q$ resonant modes, the refractive index of the cavity must be higher than that of the surrounding medium; this condition leads to the confinement of light inside the cavity due to total internal reflection. Reported $Q$-factors measured in microspheres and microtoroids range from $10^{8}$ to $10^{10}$ [6,7]. Liquid microdroplets represent alternative ultrahigh- $Q$ optical microcavities, owing to their ideally spherical geometry and smooth surface [8,9]. Droplet-based optical cavities have been subject to extensive theoretical and experimental studies. Chylek calculated the spacing between the neighboring WGMs of oil droplets and compared the theoretical results to experimental data with $\sim 1 \%$ accuracy [10]. Eversole $e t$ al. studied the spectral positions of WGMs and used them to determine the droplet refractive index [11,12]. In a number of experimental investigations, dye lasing [13-18], stimulated Raman scattering [19-22], and Raman lasing [23,24] from droplets of various liquids surrounded by air or another immiscible liquid with low index of refraction have been analyzed and characterized. At visible wavelengths, absorption-limited $Q$-factors of $\sim 10^{8}$ have been predicted for the first-order WGMs of water microdroplets in air with a size parameter $\alpha=2 \pi a / \lambda$ ( $a$ and $\lambda$ being the droplet radius and the light wavelength, respectively) larger than $\sim 60$ [25]. Experiments involving a stream of liquid microdroplets floating in air have revealed $Q$-factors up to $5 \times 10^{5}$, using measurements of the photon lifetime of light scattered elastically from the droplets [26]. In addition, experiments with cavity-enhanced energy transfer between donor and acceptor molecules have suggested $Q$-factors of $\sim 5 \times 10^{6}$ for the WGMs of single electrodynamically levitated glycerol microdroplets in air $[27,28]$. Despite these demonstrations, characterization of $Q$-factors of liquid microdroplets in air based on direct high-resolution determination of WGM linewidths has not been reported. The main experimental challenge of such measurements lies in stabilizing the droplet position and size during the measurement and simultaneously coupling efficiently the probe laser light into the droplet.

In this article, we report direct, linewidth-based measurements of ultrahigh- $Q$ factors of individual optical resonances excited in liquid microdroplets surrounded by air. The droplets were deposited on a superhydrophobic surface and their WGMs were probed using tapered optical fiber waveguides. The experimental configuration with a superhydrophobic 
surface is robust and easy to implement, and preserves the spherical shape of the microdroplets while stabilizing their position [29]. Thus, it simplifies significantly the measurements of $Q$-factors of liquid droplets in air and allows prolonged investigation of individual droplets. In our experiments, the droplet size was stabilized by adding glycerol to the aqueous droplets and keeping the droplets in a closed chamber with controlled relative humidity. We used a tapered optical-fiber waveguide to couple light from a narrowlinewidth tunable laser into an individual surface-supported droplet of glycerol-water mixture and measured the fiber transmission as a function of the laser wavelength. Tapered optical-fiber coupling is commonly used for characterizing ultrahigh- $Q$ microcavities [1-4] ]. In contrast to free-space coupling, fiber tapers allow selective phase-matched excitation of individual WGMs [30] with a coupling efficiency better than $90 \%$ [31] and a spectral resolution limited only by the linewidth of the laser used. We note that $Q$-factors of individual liquid droplets have been previously characterized using tapered fibers [32]. In that work, the studied aqueous droplets were immersed in an immiscible cladding liquid that prevented evaporation of the droplet liquid. While this approach provided stabilization of the droplet size, simultaneous requirements of liquid immiscibility and refractive index contrast put limits on the suitable droplet/immersion medium liquid pairs.

\section{EXPERIMENTAL SETUP AND PROCEDURES}

The experimental setup used for the ultrahigh- $Q$ WGM spectroscopy of surface-supported liquid microdroplets is depicted in Fig. 1. Tapered optical fiber waveguides were manufactured from a single-mode optical fiber (SM600; Thorlabs) by the heat-pull method using a stationary hydrogen flame $[33,34]$. During the taper pulling, the transmission of light from an auxiliary laser $(\lambda=637 \mathrm{~nm})$ through the formed taper was monitored and the pulling of the fiber was halted when oscillations in the transmitted light intensity ceased. Since these oscillations arose due to interference of multiple transverse modes propagating in the fiber, their disappearance indicated single-mode operation of the taper [34]. The transmission of the final tapers was typically higher than $90 \%$ and scanning electron microscope imaging revealed taper diameters in the range of 500-700 $\mathrm{nm}$. The taper was mounted on a three-axis piezo stage (Tritor 102SG; Piezosystem Jena) that allowed fine positioning of the taper with respect to the droplet with a resolution better than $2 \mathrm{~nm}$. Superhydrophobic surfaces with mean roughness on the order of $\sim 50-100 \mathrm{~nm}$ were prepared by spin-coating a suspension of hydrophobic silica nanoparticles (Aeroxide LE1, average particle size $14 \pm 3 \mathrm{~nm}$; Evonik) in ethanol on glass slides [29,35]. Individual droplets of glycerol-water mixture were deposited on the superhydrophobic surface at ambient room humidity ( $\sim 2-44 \%$ ) using a glass microcapillary connected to a syringe. The initial concentration of glycerol in the mixture was $47.8 \%$ w/w; however, this increased after the droplet deposition due to the evaporation of water in the low-humidity ambient environment. Subsequently, the surface with a single droplet and the mounted taper were enclosed in a chamber having a total volume of $\sim 1.9 \mathrm{~L}$ with its relative humidity adjusted to $53 \%$ by a saturated solution of magnesium nitrate. At this relative humidity and with an ambient temperature of
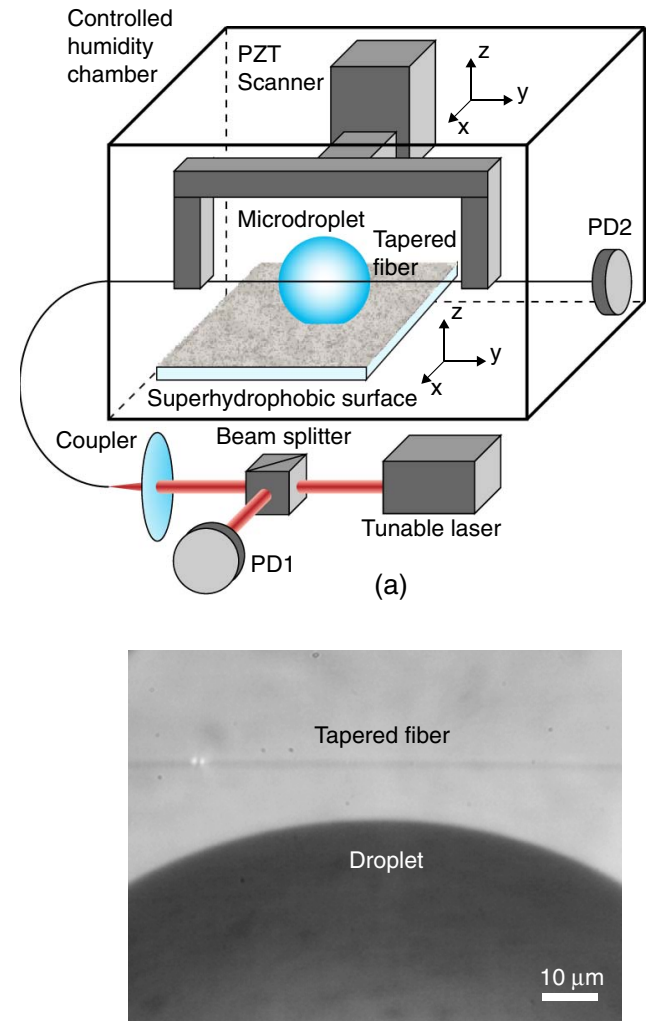

(b)

Fig. 1. (Color online) (a) Experimental setup for ultrahigh- $Q$ spectroscopy of surface-supported liquid microdroplets. PD1 (PD2), photodetector monitoring the light power at the input (output) of the tapered fiber. (b) Image of a surface-supported glycerol-water microdroplet with a tapered fiber positioned in its equatorial plane (view along the $z$-axis).

$\sim 23^{\circ} \mathrm{C}$, the equilibrium concentration of glycerol in the droplet is $77.1 \% \mathrm{w} / \mathrm{w}$ [36], which corresponds to a refractive in$\operatorname{dex} n_{D}=1.439$ [37]. The experiments were carried out within $2 \mathrm{~h}$ after the droplet deposition. During this time, the humidity in the sample chamber gradually approached the equilibrium value of $53 \%$ and the droplet size roughly stabilized. The typical equilibrium radius of droplets used in the $Q$-factor measurements ranged from $\sim 100 \mu \mathrm{m}$ to $\sim 200 \mu \mathrm{m}$. Light from a tunable external-cavity diode laser (tuning range 632.5$637 \mathrm{~nm}$, linewidth $<300 \mathrm{kHz}$, Velocity 6304; New Focus) was coupled into the tapered fiber that was then brought to the proximity of the droplet to induce coupling between the taper and the droplet. While approaching the taper to the droplet, we recorded simultaneously the taper transmission spectra and stopped approaching when we observed resonant transmission dips with a sufficient modulation depth (more than 20\%). Since efficient coupling between the taper and the droplet requires overlap of evanescent fields of the two objects, the distance between the taper and the droplet was on the order of the penetration depth of the evanescent field $d=\lambda /\left(2 \pi\left(n_{D}^{2}-1\right)^{1 / 2}\right)$ [38]. For our experimental conditions, $d \approx 100 \mathrm{~nm}$. In order to minimize the absorption of the pump laser light in the droplet liquid, we carried out the measurements in the visible part of the spectrum where both water and glycerol are virtually nonabsorbing. The laser power was monitored both at the input and at the output of the tapered fiber and the measured taper transmission was 
normalized by the input power in order to cancel out the laser power variations during wavelength tuning. Wavelength scans of the tunable probe laser were carried out at low and high resolutions. During the low-resolution scans, the position of the tuning mirror inside the laser was changed by a DC motor with a spectral resolution of $0.02 \mathrm{~nm}$ over the whole $4.5 \mathrm{~nm}$ tuning range. These low-resolution scans were used to determine the free spectral range (FSR) of the droplet cavity. Due to the DC motor actuation of the tuning mirror, the low-resolution scans were slow (scan duration 100 s). In contrast, droplet WGM spectra with the resolution limited by the laser linewidth $(<300 \mathrm{kHz})$ were acquired in the highresolution scans that were performed within the $\sim 90 \mathrm{GHz}$ mode-hop free tuning range by the displacement of the tuning mirror with a piezoelectric transducer. The calibration of wavelength tuning in the high-resolution scans was carried out using a scanning Fabry-Perot interferometer. The highresolution spectra over the full fine-tuning range of $\sim 125 \mathrm{pm}$ with the laser linewidth-limited resolution of $\sim 0.4 \mathrm{fm}$ could be acquired in approximately $2 \mathrm{~s}$.

\section{RESULTS}

\section{A. Stability of Droplet Shape and Size}

Figure 2 shows a side view of a representative glycerolwater microdroplet standing on a superhydrophobic surface. This figure illustrates the very good sphericity of the droplet shape and its high contact angle on the superhydrophobic surface (typically $>155^{\circ}$ ). We analyzed the droplet shape by locating the droplet contour in the images and fitting this contour with a circle using a custom-written routine implemented in IgorPro (WaveMetrics). From the shape analysis, we obtained the relative $\mathrm{rms}$ difference between the true droplet contour and the best-fit circle $D / a \approx 0.2 \%$, where $D$ is the absolute rms difference between the true contour and the fit and $a$ is the droplet radius. The droplet was deformed toward an
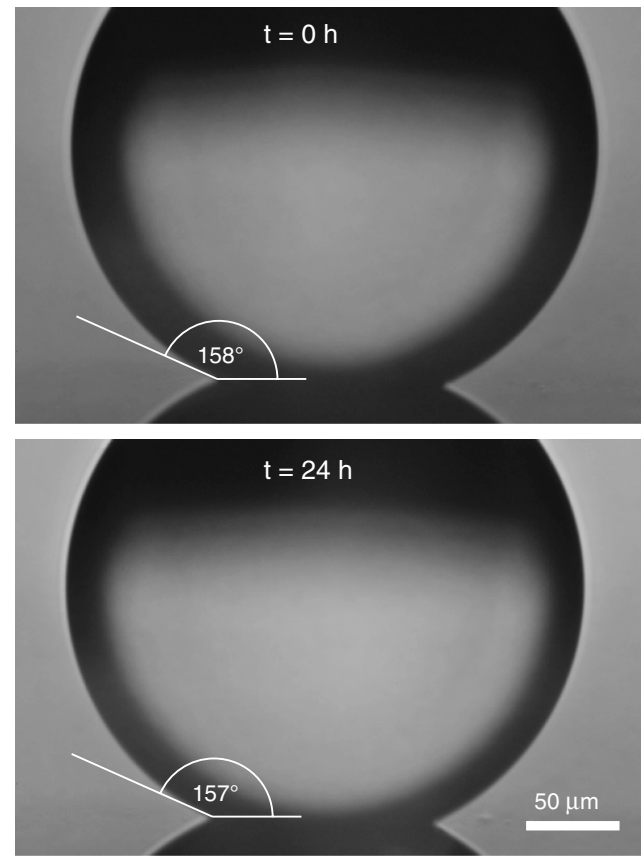

Fig. 2. Images of a glycerol-water microdroplet standing on a superhydrophobic surface (side view along the surface plane). Images were acquired at different times $t$ elapsed since the droplet generation. oblate spheroid that is consistent with gravity-induced deformation (see Section 3.B). In order to asses the stability of surface-supported droplets, we monitored the same droplet exposed to the ambient room conditions (average relative humidity $\sim 55 \%$, temperature $25^{\circ} \mathrm{C}$ ) for an extended period of time (up to 3 days). Comparison of the top and bottom images in Fig. 2 that were acquired with a time separation of $24 \mathrm{~h}$ shows that a prolonged exposure of the droplet to ambient atmosphere did not have any influence on the droplet contact angle and overall shape. The droplet shape analysis revealed a slight decrease of the droplet size in $24 \mathrm{~h}$ (relative radius change $\sim 2 \%$ ). This size change can be attributed to the fluctuations in the ambient room humidity between the acquisition of the subsequent images.

In Fig. 3, a typical low-resolution transmission spectrum of a tapered fiber waveguide coupled to a microdroplet is shown. The average optical input power coupled into the taper was approximately $60 \mu \mathrm{W}$. Despite the residual variations of the nonresonant transmission background during the wavelength scan, sharp transmission dips corresponding to the excitation of individual WGMs of the droplet cavity are clearly visible. The overall structure of the WGM spectra is rather complex with the mode degeneracy largely lifted due to a slight droplet deformation by gravity and contact line pinning on surface inhomogeneities (see also the discussion in Section 3.B). Thus, we did not attempt to assign the observed taper transmission dips to specific mode orders. Using the asymptotic formula of Chylek [39]

$$
a=\frac{\lambda^{2}}{2 \pi \mathrm{FSR}} \frac{\arctan \left(\left[n_{D}^{2}-1\right]^{1 / 2}\right)}{\left(\left[n_{D}^{2}-1\right]^{1 / 2}\right)}
$$

and assuming an effective refractive index of $n_{D}=1.439$ for the glycerol-water mixture at 53\% relative humidity, we calculated the droplet radius $a$ from the FSR of $0.414 \mathrm{~nm}$ at the mean wavelength $\lambda=635 \mathrm{~nm}$ to be $a=120 \mu \mathrm{m}$. This value matched well the radius of $\sim 127 \mu \mathrm{m}$ obtained from the droplet image analysis, considering ambiguity in defining precisely the location of the droplet boundary in the image that furthermore depends on microscope focusing. For droplets much larger than the light wavelength, the FSR given by Eq. (2) is rather insensitive to the droplet radius changes $\Delta a$ (the FSR change $\left.\Delta \mathrm{FSR} \sim-(\lambda / a)^{2} \Delta a\right)[\underline{10,39]}$. On the other

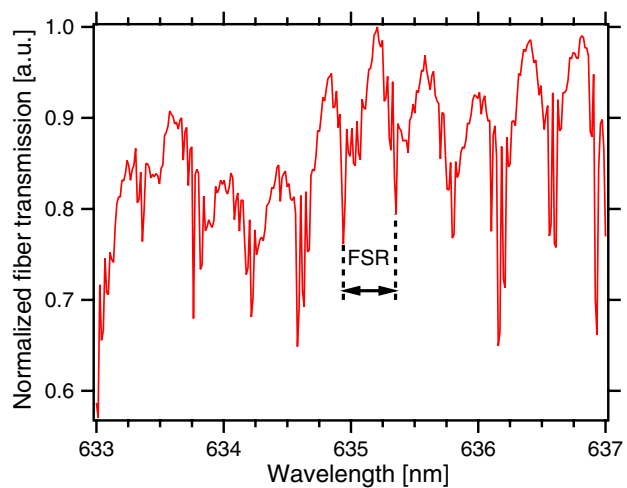

Fig. 3. (Color online) Low-resolution transmission spectrum of a tapered fiber waveguide coupled to a glycerol-water droplet in air as a function of the pump laser wavelength. Spectrum acquisition time was $\sim 100 \mathrm{~s}$. FSR indicates the free spectral range of the microdroplet cavity $(\mathrm{FSR}=0.414 \mathrm{~nm})$. 
hand, the observed shift of the absolute WGM positions $\Delta \lambda$ scales with $\Delta a$ as [5]

$$
\Delta \lambda=\left(\frac{\lambda}{a}\right) \Delta a .
$$

Consequently, at $\lambda=635 \mathrm{~nm}$ and $a=120 \mu \mathrm{m}$, even a modest resolution of $\Delta \lambda=0.1 \mathrm{~nm}$ in detecting the WGM position corresponds to the smallest resolvable change of the droplet radius $\Delta a=18.9 \mathrm{~nm}$, which is well below the typical effective pixel size of CCD detectors used in high-resolution optical microscopy ( $\sim 65 \mathrm{~nm}$, assuming physical pixel size $6.45 \mu \mathrm{m}$ and 100-times optical magnification [40]). Hence, tracking of the absolute position of a selected WGM provides much more precise information on the droplet size changes than the analysis of the droplet image. For this reason, all quantifications of the droplet size changes in the following text are based on the WGM position tracking.

Repeated high-resolution wavelength scans of the taper transmission revealed spectral shifting of the observed WGM structure that indicated changes of the droplet size. In Figs. 4(a) and 4(b), an illustration of the WGM structure drifting as a whole to the blue end of the spectrum is given.
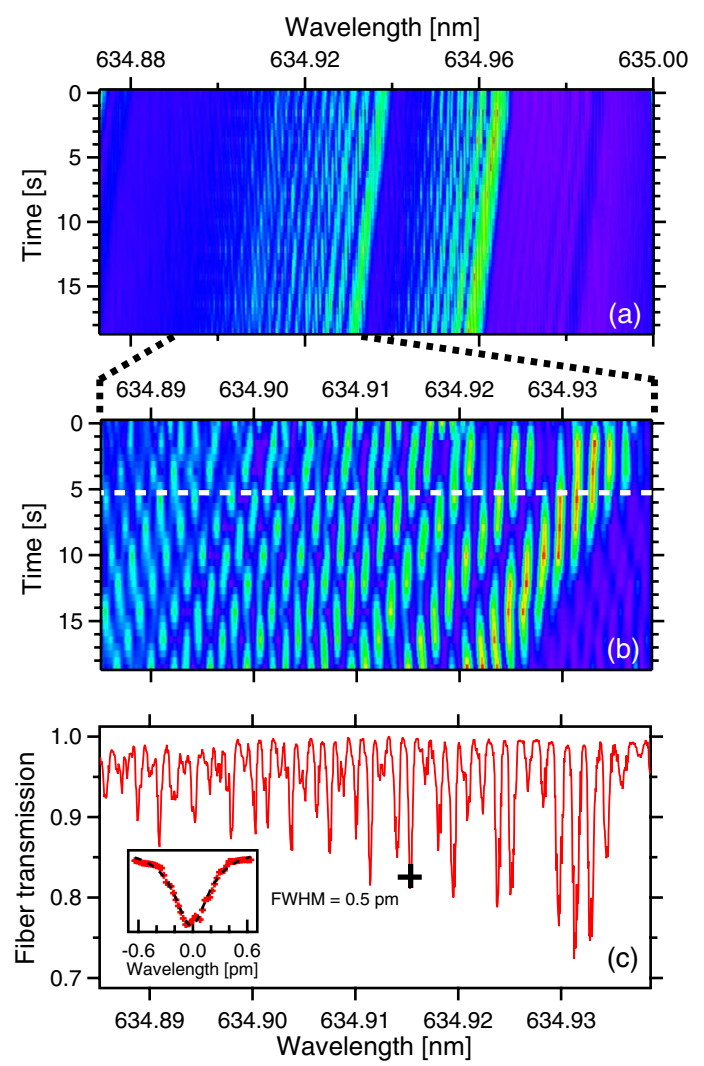

Fig. 4. (Color online) High-resolution transmission spectrum of a tapered fiber waveguide coupled to a glycerol-water droplet in air as a function of the pump laser wavelength and time. (a) Time series of transmission scans over the whole fine-tuning range of the laser (spectral interval width $\sim 125 \mathrm{pm}$ ). (b) Time series of transmission scans over the tuning range occupied by a group of WGMs shown in the left part of spectral map (a) (spectral interval width $\sim 54 \mathrm{pm}$ ). (c) Spectral profile of the tapered fiber transmission along the dashed white line shown in spectral map (b). Black cross denotes a representative WGM used for the $Q$-factor analysis (see graph inset and text). Average droplet radius was $130 \mu \mathrm{m}$ and rate of the droplet radius change was $-0.17 \mathrm{~nm} / \mathrm{s}$.
Here, blue-shifting of the modes indicates decreasing size of the droplet that is accompanied by shortening of the resonant modal path in the droplet; correspondingly, red-shifting of the modes is the sign of the droplet size increase. In general, the droplet radius evolves with time as a result of a complex interplay between evaporation and condensation processes and, thus, the size of the loaded droplet can both decrease and increase. The dynamics of changes of the droplet size then depend on the total probe power coupled into the droplet and deviations from the thermodynamic equilibrium between the liquid and gas phases in the humidity chamber. For the example spectral recording presented in Fig. 4, the rate of the droplet radius change determined from Eq. (3) for the measured spectral drift of $-0.83 \mathrm{pm} / \mathrm{s}$ and the average droplet radius of $130 \mu \mathrm{m}$ was $-0.17 \mathrm{~nm} / \mathrm{s}$. Since our experiments were typically carried out on the time scale of $\sim 2 \mathrm{~h}$, the above rate of the droplet size change corresponds to the relative droplet size stability of $\sim 0.9 \%$ over the duration of an experiment.

\section{B. Lifting of Azimuthal Mode Degeneracy}

A spherical resonant cavity with radius $a$ and refractive index $n_{s}$ can host WGMs characterized by a radial mode number $n$, an angular mode number $l$, and an azimuthal mode number $m= \pm l, \pm(l-1), \ldots, 0$. For a perfect sphere, the azimuthal modes $m$ corresponding to a given $l$ are spectrally $(2 l+1)$ degenerate. Deformation of the sphere toward a spheroid with polar and equatorial radii $r_{p}, r_{e}$, respectively, leads to the lifting of the azimuthal mode degeneracy. Resonant frequency of the $m$ th mode is then given by $[\underline{41}, \underline{42}]$ :

$$
\omega(m)=\omega_{0}\left(1-\frac{e}{6}\left[1-\frac{3 m^{2}}{l(l+1)}\right]\right) .
$$

Here, $\omega_{0}$ is the frequency of the original degenerate mode of the unperturbed sphere and the eccentricity of the spheroid is $e=\left(r_{p}-r_{e}\right) / a$. The value of $e$ is positive for a prolate spheroid and negative for an oblate spheroid.

The equilibrium shape of droplets deposited on a superhydrophobic surface is the result of the interplay between the forces of surface tension and gravity. In general, sessile droplets tend to be flattened by gravity, and for small deformations the droplet shape is well approximated by an oblate spheroid [43]. Besides gravity, the droplet shape can be perturbed by other factors, the most prominent of which is inhomogeneity of the superhydrophobic surface that causes pinning and distortion of the droplet contact line. Consequently, the droplet shape departs from that of a perfect sphere or a rotationally symmetrical spheroid. All additional distortions that increase the droplet eccentricity lead to further frequency splitting of the azimuthal $m$-modes.

Droplet eccentricity $e$ can be estimated from the measured mode frequency splitting. From Eq. (4), the frequency difference $\Delta \omega(m)$ between two neighboring $m$-modes is

$$
\Delta \omega(m)=\omega(m+1)-\omega(m)=\frac{\omega_{0} e}{l(l+1)}\left[m+\frac{1}{2}\right] .
$$

For a glycerol-water droplet with radius $a=130 \mu \mathrm{m}$ and refractive index $n_{s}=1.44$ probed with a mean laser wavelength $\lambda=635 \mathrm{~nm}$, the approximate value of $l$ is 


$$
l \approx \frac{2 \pi a n_{s}}{\lambda} \approx 1852 \gg 1
$$

Moreover, in our experimental geometry (excitation of droplet WGMs via a tapered fiber located at the droplet equatorial plane), we excite most efficiently the WGMs with $|m| \approx l$ and the intensity of the observed WGMs falls off rapidly with increasing value of $(l-|m|)$ [32]. Hence, Eq. (ㅁ) simplifies to

$$
\Delta \omega(m) \approx \frac{\omega_{0} e}{l^{2}}\left[m+\frac{1}{2}\right] \approx \frac{\omega_{0} e}{l},
$$

and, upon substituting Eq. (ㅁ) into Eq. ()), we obtain

$$
e \approx \frac{\Delta \omega(m)}{\omega_{0}} \frac{2 \pi a n_{s}}{\lambda}=\frac{2 \pi a n_{s} \Delta \lambda}{\lambda^{2}},
$$

where $\Delta \lambda$ is the measured mode splitting. For the spectrum presented in Fig. 4(c), the spacing of neighboring modes is $\approx 1.5 \mathrm{pm}$. Because of the mode excitation symmetry (the taper located in the droplet equatorial plane), odd azimuthal modes are not excited and the observed mode separation is actually equal to $2 \Delta \lambda$ [32]. Therefore, $\Delta \lambda \approx 0.75 \mathrm{pm}$ and the droplet eccentricity $e \approx 2.2 \times 10^{-3}$. This value of eccentricity is comparable to the rms droplet shape distortion of $\sim 2 \times 10^{-3}$ that was obtained from the droplet shape analysis presented in Section 3.A.

\section{C. $O$-Factor Measurements}

In order to characterize the $Q$-factors of WGMs of surfacesupported liquid microdroplets, we conducted series of relatively fast, high-resolution spectral scans over a narrow spectral range of $54 \mathrm{pm}$ within $2.06 \mathrm{~s}$. In Fig. $4(\mathrm{c})$, an example of such high-resolution spectral recording is presented for a group of WGMs collectively drifting to the blue end of the spectrum. This group of WGMs consists of an intense long-wavelength mode accompanied by a number of shorterwavelength modes whose intensities decrease with decreasing wavelength. Such a spectrum is consistent with a family of WGMs with the same angular and radial mode numbers and different azimuthal mode numbers originating from the degeneracy lifting in a sphere deformed toward an oblate spheroid (see Section 3.B). Here, the longest-wavelength mode corresponds to the highest absolute value of the azimuthal mode number that belongs to the modes circulating in the droplet equatorial plane [41,42]. In accordance with Eq. (2), the relative positions of individual WGMs are virtually unaffected by the droplet size drift. A Lorentzian fit of a representative WGM denoted by a black cross in Fig. 4(c) gave the mode FWHM $\delta \lambda=0.5 \mathrm{pm}$. Knowing $\delta \lambda$, the $Q$-factor at the mean laser wavelength $\lambda=635 \mathrm{~nm}$ could be determined using Eq. (1). Since the droplet size changed slightly during the spectrum acquisition, the $Q$-factor should be calculated after correcting the measured FWHM for the spectral drift of WGMs. Our highresolution spectral scans were carried out from red to blue wavelengths; thus, for a blue-drifting mode, the true FWHM of the mode is smaller than the measured one. Taking into account the measured rate of the WGM position drift of $\sim 0.8 \mathrm{pm} / \mathrm{s}$ and the spectral acquisition rate of $54 \mathrm{pm} / 2.06 \mathrm{~s} \approx$ $26.2 \mathrm{pm} / \mathrm{s}$, we obtain the true FWHM of $\sim 0.485 \mathrm{pm}$ and, consequently, the $Q$-factor of $1.31 \times 10^{6}$ for the selected WGM.

Images of a glycerol-water microdroplet on a superhydrophobic surface presented in Fig. 2 suggest that the droplet

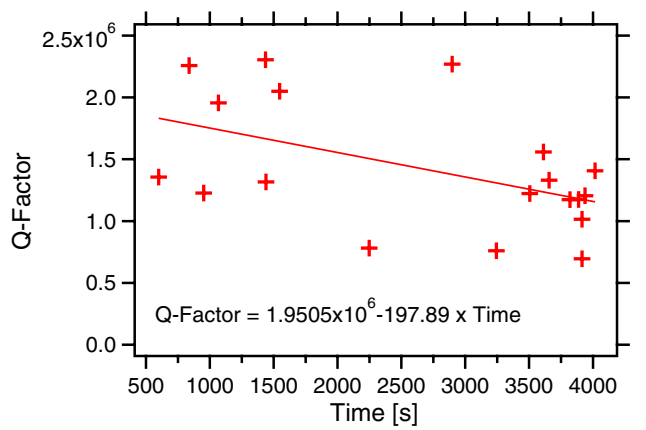

Fig. 5. (Color online) Time evolution of the $Q$-factor of degeneracylifted azimuthal WGMs of a surface-supported microdroplet. Time $t=0 \mathrm{~s}$ corresponds to the droplet deposition on the surface. Crosses represent experimental data, line is the linear fit of this data. Average droplet radius was $150 \mu \mathrm{m}$.

shape and contact angle are stable on the time scale of hours. This implies that the optical properties of surface-supported microdroplet cavities should not change significantly after the droplet deposition. In Fig. 5, time evolution of the $Q$-factor of a single microdroplet is presented within the interval of $\sim 1 \mathrm{~h}$ following the droplet deposition. Values of $Q$-factors were determined using the above-described procedure. In order to minimize the possibility of forming a liquid bridge between the tapered fiber and the droplet, the taper was repeatedly moved toward and away from the droplet between consecutive $Q$-factor measurements. Therefore, it is likely that modes with different angular and azimuthal numbers were characterized in individual measurements shown in Fig. 5. As illustrated by this figure, the characteristic $Q$-factors of degeneracy-lifted azimuthal WGMs of the droplet decreased slightly with time. While we did not observe any discernible changes in the droplet shape over several hours, it is likely that contaminant particles originating from the droplet liquid and/or slowly dissolving superhydrophobic surface accumulated at the liquid-air interface during this time. Such accumulation of contaminant particles would then lead to an increase in the light scattering at the droplet surface and, thus, increased loss of the cavity that is equivalent to a lower $Q$-factor. It is worth noting that it is not possible to observe directly binding of individual nanoparticles forming the superhydrophobic surface to the droplet since the mass of nanoparticles ( 3.8 attogram/ particle) is too small to cause a detectable shift of the WGM positions [44]. Hence, accumulation of nanoparticles on the droplet surface manifests itself as a continuous process rather than a sequence of discrete jumps of the WGM properties. In addition, unlike with solid cavities, contaminant particles on liquid surfaces are not immobilized but instead diffuse in and out of the WGM field, which further smears the optical response of the cavity. Despite the slight decrease with time, the droplet $Q$-factor remains on the order of $10^{6}$. Consistence of this value with the expected experimental limits on the measured $Q$-factors is discussed in the next section.

\section{EXPERIMENTAL LIMITS ON MEASURED $Q$-FACTORS}

\section{A. Contributions to Overall $Q$-Factor of a Resonant Cavity}

In general, the overall quality factor $Q$ defined by Eq. (1) consists of several components: 


$$
\frac{1}{Q}=\frac{1}{Q_{\mathrm{rad}}}+\frac{1}{Q_{\mathrm{mat}}}+\frac{1}{Q_{\mathrm{s.s}}},
$$

where $1 / Q_{\text {rad }}$ characterizes losses of the resonant cavity due to radiation, $1 / Q_{\text {mat }}$ describes losses due to material absorption and bulk scattering, and $1 / Q_{\text {s.s. }}$ is the contribution of scattering from surface inhomogeneities [5,6]. In perfect spheres with $2 a / \lambda \geq 15$, radiative quality factor $Q_{\mathrm{rad}}>10^{11}$ [6]. In spheroids characterized by eccentricity $e$, photon paths generally depart from ideal circularity. This results in a lowering of $Q_{\text {rad }}$ relative to undeformed spheres that is proportional to $e^{2}$ and negligible for WGMs with modal paths localized in the equatorial plane of the spheroid [45]. As these are the modes we predominantly excite and characterize in our experimental geometry, we can assume that the value of $Q_{\text {rad }}$ is approximately unaltered by the droplet deformation. Material-related quality factor $Q_{\text {mat }}$ represents typically the main limit for the overall $Q$. This contribution can be expressed as

$$
Q_{\mathrm{mat}}=\frac{2 \pi n_{s}}{\alpha \lambda}
$$

where $\alpha$ is the linear absorption coefficient of the cavity material and $n_{s}$ is the cavity refractive index at wavelength $\lambda$. For a glycerol-water mixture at $\lambda=635 \mathrm{~nm}, \alpha \approx 2.8 \times$ $10^{-3} \mathrm{~cm}^{-1}$ [46] and $n_{s}=1.44$ [37], and, thus, $Q_{\text {mat }} \approx 5 \times 10^{7}$. Finally, $Q_{\text {s.s. }}$ can be expressed as

$$
Q_{\mathrm{s.s.}}=\frac{\lambda^{2} a}{\pi^{2} \Delta^{2} B},
$$

with $\Delta$ being the rms size of surface inhomogeneities and $B$ their correlation length [6]. For glycerol-water droplets, thermal fluctuations of the droplet shape lead to $\Delta \approx 0.07 \mathrm{~nm}$ [see Eq. (12)] and $B$ can be estimated from the characteristic wavenumber $q$ of the maximal liquid surface roughness $\left(q \sim 10^{7} \mathrm{~cm}^{-1}\right)$ as $B \approx 2 \pi / q \approx 6.3 \mathrm{~nm}$ [47]. Assuming further that the droplet radius $a=130 \mu \mathrm{m}, \overline{Q_{\text {s.s. }}} \approx 1.7 \times 10^{11}$.

From the above analysis, it follows that the expected limit on the intrinsic value of $Q$-factor measured in our experiments is set by $Q_{\text {mat }}$ at the level of $\approx 5 \times 10^{7}$. This limit, however, is approximately 10 times larger than the maximum observed $Q$ factors. As argued in Section 4.B, we attribute this difference to thermally induced droplet shape fluctuations that lower the $Q$-factor values measured with the use of tapered optical fibers.

\section{B. Fluctuation of Mode Position Due to Thermally Induced Droplet Shape Fluctuations}

Due to thermal excitation, droplet shape undergoes random dynamic distortions with a characteristic amplitude given by [두]:

$$
\Delta=\left[\frac{k_{B} T}{4 \pi \gamma}\right]^{1 / 2},
$$

where $k_{B}$ is the Boltzmann constant, $T$ is the thermodynamic temperature, and $\gamma$ is the surface tension of the droplet liquid. For a glycerol droplet with $\gamma=64 \mathrm{mN} / \mathrm{m}$ at room temperature $T=293^{\circ} \mathrm{K}, \Delta \approx 0.07 \mathrm{~nm}$.

According to Eq. (4), the frequency $\omega(m)$ of the $m$ th azimuthal mode depends linearly on the droplet eccentricity $e$. Thus, a change in $e$ leads to a corresponding change in $\omega(m)$ :

$$
d \omega(m)=-\frac{\omega_{0}}{6}\left[1-\frac{3 m^{2}}{l(l+1)}\right] d e .
$$

Assuming droplet volume conservation upon deformation into a spheroid (i.e. $r_{p} r_{e}^{2}=a^{3}$ ), a shape distortion $\Delta$ in the direction of the droplet polar radius $r_{p}$ is associated with a change in eccentricity $\delta e$ :

$$
\delta e=\left[1+\frac{1}{2}\left(\frac{a}{r_{p}}\right)^{3 / 2}\right] \frac{\Delta}{a} .
$$

For glycerol-water microdroplets supported by a superhydrophobic surface, the droplet distortion from sphericity is very small $\left(e \approx 2.2 \times 10^{-3}\right)$ and also $\Delta / a \ll 1$. Thus, $r_{p} \approx a$ and

$$
\delta e \approx \frac{3}{2} \frac{\Delta}{a} .
$$

Upon substituting Eq. (15) into Eq. (13), we obtain a relative frequency spread of the $m$ th mode position as

$$
\frac{d \omega(m)}{\omega_{0}}=-\frac{1}{6}\left[1-\frac{3 m^{2}}{l(l+1)}\right] \frac{3}{2} \frac{\Delta}{a} .
$$

Assuming again $l \gg 1$ and $|m| \approx l$, this simplifies to

$$
\frac{d \omega(m)}{\omega_{0}}=\frac{1}{2} \frac{\Delta}{a} .
$$

For the considered values of $\Delta=0.07 \mathrm{~nm}$ and $a=130 \mu \mathrm{m}$, $d \omega(m) / \omega_{0} \approx 2.7 \times 10^{-7}$. As the amplitude $\Delta$ of the thermally induced droplet shape fluctuations is much smaller than the static droplet deformation due to gravity and contact line pinning $\Delta_{g} \approx e a \approx 286 \mathrm{~nm}$, degeneracy-lifted azimuthal modes of thermally vibrating droplets remain well resolved and do not mix with each other [항.

Thermal fluctuations of the droplet shape typically occur in the frequency range of $\sim 10^{5} \mathrm{~Hz}$. On the other hand, spectroscopic characterization of the droplet WGMs using a tapered fiber and a narrow-linewidth tunable laser was carried out on the time scale of $\sim 1 \mathrm{~s}$. Hence, the spectral measurement detects an "effective" width of the fluctuating WGM given by Eq. (17). This in turn corresponds to an effective quality factor $Q_{\mathrm{eff}}$

$$
Q_{\text {eff }}=\frac{\omega_{0}}{d \omega(m)}=\frac{2 a}{\Delta} \approx 3.7 \times 10^{6} .
$$

It is this value of $Q_{\text {eff }}$ (and not the intrinsic quality factor of the modes set by the value of $Q_{\text {mat }}$ in our system) that represents an upper limit on the quality factors measurable with the current experimental setup. This finding is consistent with the experimental data presented in Figs. $\underline{4}$ and $\underline{5}$.

\section{CONCLUSION}

In this article, we have presented systematic experimental characterization of ultrahigh- $Q$ resonant modes (WGMs) of liquid microdroplets surrounded by air. To this end, we have deposited the droplets of the glycerol-water mixture on superhydrophobic surfaces with high contact angles and used light coupling from a tapered optical fiber waveguide to 
record the droplet WGM spectra. The measured $Q$-factors exceeded $10^{6}$ and were stable on the time scale of $1 \mathrm{~h}$. We have shown that slight residual deformations of the droplet shape due to the presence of the superhydrophobic surface with the contact angle smaller than $180^{\circ}$ do not represent a restriction in the ultrahigh- $Q$ spectroscopic measurements. Despite the high experimental values of the $Q$-factor, we have not reached the fundamental limit set by the light absorption in the droplets; our measurements have been limited by the thermally induced shape fluctuations of the droplets. Even though the stability of the droplet size in air has been greatly improved by controlling the humidity of the ambient atmosphere, some residual drift of the droplet radius has been observed. This issue can be further improved by active photothermal control of the droplet size [36]. The experimental configuration involving a superhydrophobic surface has allowed for the first time prolonged spectroscopic measurements on individual liquid droplets in air and simplified significantly efficient phase-matched light coupling into the droplets. Hence, it represents a well-suited platform for characterizing liquidbased micro-optical components.

\section{ACKNOWLEDGMENTS}

This work was partially supported by TÜBİTAK (Grant No. 109T734), the European Commission Marie Curie IEF (Contract No. PIEF-GA-2009-252579; A.J.), the Alexander von Humboldt Foundation, and a FABED Young Investigator Research Award (A.K.)

\section{REFERENCES}

1. K. J. Vahala, "Optical microcavities," Nature 424, 839-846 (2003).

2. T. J. Kippenberg and K. J. Vahala, "Cavity opto-mechanics," Opt. Express 15, 17172-17205 (2007).

3. A. M. Armani, R. P. Kulkarni, S. E. Fraser, R. C. Flagan, and K. J. Vahala, "Label-free, single-molecule detection with optical microcavities," Science 317, 783-787 (2007).

4. F. Vollmer and S. Arnold, "Whispering-gallery-mode biosensing: label-free detection down to single molecules," Nat. Methods 5, 591-596 (2008).

5. G. C. Righini, Y. Dumeige, P. Feron, M. Ferrari, G. N. Conti, D. Ristic, and S. Soria, "Whispering gallery mode microresonators: fundamentals and applications," Rivista Nuovo Cimento 34, 435-488 (2011).

6. M. L. Gorodetsky, A. A. Savchenkov, and V. S. Ilchenko, "Ultimate $Q$ of optical microsphere resonators," Opt. Lett. 21, 453-455 (1996)

7. D. K. Armani, T. J. Kippenberg, S. M. Spillane, and K. J. Vahala, "Ultra-high- $Q$ toroid microcavity on a chip," Nature 421, 925-928 (2003).

8. V. V. Datsyuk, "Optics of microdroplets," J. Mol. Liq. 84, 1308-1316 (2001)

9. J. P. Reid and L. Mitchem, "Laser probing of single-aerosol droplet dynamics," Annu. Rev. Phys. Chem. 57, 245-271 (2006).

10. P. Chylek, "Resonance structure of Mie scattering: distance between resonances,” J. Opt. Soc. Am. A 7, 1609-1613 (1990).

11. J. D. Eversole, H.-B. Lin, and A. J. Campillo, "Cavity-mode identification of fluorescence and lasing in dye-doped microdroplets," Appl. Opt. 31, 1982-1991 (1992).

12. J. D. Eversole, H.-B. Lin, A. L. Huston, A. J. Campillo, P. T. Leung, S. Y. Liu, and K. Young, "High-precision identification of morphology-dependent resonances in optical processes in microdroplets,” J. Opt. Soc. Am. B 10, 1955-1968 (1993).

13. S.-X. Qian, J. B. Snow, H. M. Tzeng, and R. K. Chang, "Lasing droplets: highlighting the liquid-air interface by laser emission," Science 231, 486-488 (1986).
14. H.-B. Lin, A. L. Huston, B. L. Justus, and A. J. Campillo, "Some characteristics of a droplet whispering-gallery-mode laser," Opt. Lett. 11, 614-616 (1986).

15. H. B. Lin, J. D. Eversole, and A. J. Campillo, "Spectral properties of lasing microdroplets," J. Opt. Soc. Am. B 9, 43-50 (1992).

16. A. Kiraz, A. Sennaroglu, S. Doganay, M. A. Dundar, A. Kurt, H. Kalaycioglu, and A. L. Demirel, "Lasing from single, stationary, dye-doped glycerol/water microdroplets located on a superhydrophobic surface," Opt. Commun. 276, 145-148 (2007).

17. M. Tanyeri, R. Perron, and I. M. Kennedy, "Lasing droplets in a microfabricated channel," Opt. Lett. 32, 2529-2531 (2007).

18. S. K. Y. Tang, Z. Li, A. R. Abate, J. J. Agresti, D. A. Weitz, D. Psaltis, and G. M. Whitesides, "A multi-color fast-switching microfluidic droplet dye laser," Lab Chip 9, 2767-2771 (2009).

19. J. B. Snow, S.-X. Qian, and R. K. Chang, "Stimulated Raman scattering from individual water and droplets at morphologydependent resonances," Opt. Lett. 10, 37-39 (1985).

20. H. B. Lin, J. D. Eversole, and A. J. Campillo, "Continuous-wave stimulated Raman scattering in microdroplets," Opt. Lett. 17, 828-830 (1992)

21. R. J. Hopkins, L. Mitchem, A. D. Ward, and J. P. Reid, "Control and characterisation of a single aerosol droplet in a single-beam gradient-force optical trap," Phys. Chem. Chem. Phys. 6, 49244927 (2004).

22. R. Symes, R. M. Sayer, and J. P. Reid, "Cavity enhanced droplet spectroscopy: principles, perspectives and prospects," Phys. Chem. Chem. Phys. 6, 474-487 (2004).

23. A. Sennaroglu, A. Kiraz, M. A. Dündar, A. Kurt, and A. L. Demirel, "Raman lasing near $630 \mathrm{~nm}$ from stationary glycerol-water microdroplets on a superhydrophobic surface," Opt. Lett. 32, 2197-2199 (2007).

24. Y. Karadag, M. Gündoğan, M. Y. Yüce, H. Cankaya, A. Sennaroglu, and A. Kiraz, "Prolonged Raman lasing in sizestabilized salt-water microdroplets on a superhydrophobic surface," Opt. Lett. 35, 1995-1997 (2010).

25. A. Serpengüzel, J. C. Swindal, R. K. Chang, and W. P. Acker, "Two-dimensional imaging of sprays with fluorescence, lasing, and stimulated Raman scattering," Appl. Opt. 31, 3543-3551 (1992).

26. J.-Z. Zhang, D. H. Leach, and R. K. Chang, "Photon lifetime within a droplet: temporal determination of elastic and stimulated Raman scattering," Opt. Lett. 13, 270-272 (1988).

27. S. Arnold and L. M. Folan, "Energy transfer and the photon lifetime within an aerosol particle," Opt. Lett. 14, 387-389 (1989).

28. P. T. Leung and K. Young, "Theory of enhanced energy transfer in an aerosol particle," J. Chem. Phys. 89, 2894-2899 (1988)

29. A. Kiraz, A. Kurt, M. A. Dündar, and A. L. Demirel, "Simple largely tunable optical microcavity," Appl. Phys. Lett. 89, 081118 (2006).

30. J. C. Knight, G. Cheung, F. Jacques, and T. A. Birks, "Phasematched excitation of whispering-gallery-mode resonances by a fiber taper,” Opt. Lett. 22, 1129-1131 (1997).

31. M. Cai, O. Painter, and K. J. Vahala, "Observation of critical coupling in a fiber taper to a silica-microsphere whispering-gallery mode system," Phys. Rev. Lett. 85, 74-77 (2000).

32. M. Hossein-Zadeh and K. J. Vahala, "Fiber-taper coupling to whispering-gallery modes of fluidic resonators embedded in a liquid medium," Opt. Express 14, 10800-10810 (2006).

33. T. A. Birks and Y. W. Li, "The shape of fiber tapers," J. Lightwave Technol. 10, 432-438 (1992).

34. F. Orucevic, V. Lefevre-Seguin, and J. Hare, "Transmittance and near-field characterization of sub-wavelength tapered optical fibers," Opt. Express 15, 13624-13626 (2007).

35. A. Jonas, Y. Karadag, N. Tasaltin, I. Kucukkara, and A. Kiraz, "Probing microscopic wetting properties of superhydrophobic surfaces by vibrated micrometer-sized droplets," Langmuir 27, 2150-2154 (2011).

36. A. Kiraz, Y. Karadağ, and M. Muradoğlu, "Large spectral tuning of a water/glycerol microdroplet by a focused laser: characterization and modeling," Phys. Chem. Chem. Phys. 10, 6446-6454 (2008).

37. D. R. Lide, CRC Handbook of Chemistry and Physics, Internet Version 2012 (CRC, 2012).http://www.hbcpnetbase.com 
38. M. Born and E. Wolf, Principles of Optics: Electromagnetic Theory of Propagation, Interference and Diffraction of Light, 7th ed. (Cambridge University, 1999).

39. P. Chylek, "Partial-wave resonances and the ripple structure in the Mie normalized exctinction cross section," J. Opt. Soc. Am. 66, 285-287 (1976).

40. J. C. Waters, "Accuracy and precision in quantitative fluorescence microscopy,” J. Cell Biol. 185, 1135-1148 (2009).

41. H. M. Lai, P. T. Leung, K. Young, P. W. Barber, and S. C. Hill, "Time-independent perturbation for leaking electromagnetic modes in open systems with application to resonances in microdroplets," Phys. Rev. A 41, 5187-5198 (1990).

42. G. Chen, M. M. Mazumder, Y. R. Chemla, A. Serpengüzel, R. K. Chang, and S. C. Hill, "Wavelength variation of laser emission along the entire rim of slightly deformed microdroplets," Opt. Lett. 18, 1993-1995 (1993).

43. V. A. Lubarda and K. A. Talke, "Analysis of the equilibrium droplet shape based on an ellipsoidal droplet model," Langmuir 27, 10705-10713 (2011).
44. S. Arnold, R. Ramjit, D. Keng, V. Kolchenko, and I. Teraoka, "Microparticle photophysics illuminates viral bio-sensing," Faraday Disc. Chem. Soc. 137, 65-83 (2008).

45. H. M. Lai, C. C. Lam, P. T. Leung, and K. Young, "Effect of perturbations on the widths of narrow morphology-dependent resonance in Mie scattering," J. Opt. Soc. Am. B 8, 1962-1973 (1991).

46. G. M. Hale and M. R. Querry, "Optical constants of water in the $200 \mathrm{~nm}$ to $200 \mathrm{um}$ wavelength region,” Appl. Opt. 12, 555-563 (1973).

47. L. F. Phillips, "A geometrical explanation for the enhanced small-scale roughness of a liquid surface," J. Phys. Chem. B 108, 1986-1991 (2004).

48. H. M. Lai, P. T. Leung, and K. Young, "Limitations on the photon storage lifetime in electromagnetic resonances of highly transparent microdroplets," Phys. Rev. A 41, 5199-5204 (1990). 